\title{
A Fixed Point Theorem on Four Complete Metric Spaces
}

\author{
Vishal Gupta, Naveen Mani, and Ashima Kanwar
}

\begin{abstract}
Our object in this paper to discuss about fixed point theorem in four metric spaces. Here we established a fixed point theorem in four complete metric spaces, which generalized many results of many authors [1]-[5].
\end{abstract}

Index Terms-Complete metric space, fixed point.

\section{INTRODUCTION}

Let $\left(X_{1}, d_{1}\right),\left(X_{2}, d_{2}\right),\left(X_{3}, d_{3}\right)$ and $\left(X_{4}, d_{4}\right)$ be complete metric spaces.

If $A_{1}: X_{1} \rightarrow X_{2}, A_{2}: X_{2} \rightarrow X_{3}, A_{3}: X_{3} \rightarrow X_{4}$ and

$A_{4}: X_{4} \rightarrow X_{1}$ are mapping than we denote

$$
M_{1}\left(x^{1}, x^{2}\right)=\left\{d_{1}^{p}\left(x^{1}, A_{4} A_{3} A_{2} x^{2}\right), d_{1}^{p}\left(x^{1}, A_{4} A_{3} A_{2} A_{1} x^{1}\right), d_{2}^{p}\left(x^{2}, A_{1} x^{1}\right)\right\}
$$

$M_{2}\left(x^{2}, x^{3}\right)=\left\{d_{2}^{p}\left(x^{2}, A_{1} A_{4} A_{3} x^{3}\right), d_{2}^{p}\left(x^{2}, A_{1} A_{4} A_{3} A_{2} x^{2}\right), d_{3}^{p}\left(x^{3}, A_{2} x^{2}\right)\right\}$

$M_{3}\left(x^{3}, x^{4}\right)=\left\{d_{3}^{p}\left(x^{3}, A_{2} A_{1} A_{4} x^{4}\right), d_{3}^{p}\left(x^{3}, A_{2} A_{1} A_{4} A_{3} x^{3}\right), d_{4}^{p}\left(x^{4}, A_{3} x^{3}\right)\right\}$

$M_{4}\left(x^{4}, x^{1}\right)=\left\{d_{4}^{p}\left(x^{4}, A_{3} A_{2} A_{1} x^{1}\right), d_{4}^{p}\left(x^{4}, A_{3} A_{2} A_{1} A_{4} x^{4}\right), d_{5}^{p}\left(x^{5}, A_{4} x^{4}\right)\right\}$

Let $F:[0, \infty] \rightarrow R^{+}$be continuous mappings in 0 with $F(0)=0$

\section{MAIN RESUlt}

\section{Theorem}

Let $\left(X_{1}, d_{1}\right),\left(X_{2}, d_{2}\right),\left(X_{3}, d_{3}\right),\left(X_{4}, d_{4}\right)$ and $\left(X_{5}, d_{5}\right)$ be complete metric spaces where. If $A_{1}: X_{1} \rightarrow X_{2}, A_{2}: X_{2} \rightarrow X_{3}, A_{3}: X_{3} \rightarrow X_{4}$ $A_{4}: X_{4} \rightarrow X_{1}$ are mapping satisfying the following

inequalities.

$$
d_{1}^{p}\left(A_{4} A_{3} A_{2} x^{2}, A_{4} A_{3} A_{2} A_{1} x^{1}\right) \leq c \max M_{1}\left(x^{1}, x^{2}\right)+F\left(\min M_{1}\right)
$$

Manuscript received March 14, 2012; revised April 23, 2012.

Vishal Gupta and Naveen Mani are with Department of Mathematics, M.M.University, Mullana, Ambala, India (e-mail address: vishal.gmn@gmail.com).

A. Kanwar is with Research Scholar, Department of Mathematics, M. M. University, Mullana, Ambala, India.

$$
d_{2}^{p}\left(A_{1} A_{4} A_{3} x^{3}, A_{1} A_{4} A_{3} A_{2} x^{2}\right) \leq c \max M_{2}\left(x^{2}, x^{3}\right)+F\left(\min M_{2}\right)
$$

$d_{3}^{p}\left(A_{2} A_{1} A_{4} x^{4}, A_{2} A_{1} A_{4} A_{3} x^{3}\right) \leq c \max M_{3}\left(x^{3}, x^{4}\right)+F\left(\min M_{3}\right)$

$d_{4}^{p}\left(A_{3} A_{2} A_{1} x^{1}, A_{3} A_{2} A_{1} A_{4} x^{4}\right) \leq c \max M_{4}\left(x^{4}, x^{1}\right)+F\left(\min M_{4}\right)$

$\forall x^{1} \in X_{1}, x^{2} \in X_{2}, x^{3} \in X_{3}, x^{4} \in X_{4}$, where, $0 \leqslant c<1$. Then $A_{5} A_{4} A_{3} A_{2} A_{1}$ has a unique fixed point $\beta_{1} \in X_{1} ; A_{1} A_{5} A_{4} A_{3} A_{2}$ has a unique fixed point $\beta_{2} \in X_{2}$; $A_{2} A_{1} A_{5} A_{4} A_{3}$ has a unique fixed point $\beta_{3} \in X_{3} ; A_{3} A_{2} A_{1} A_{5} A_{4}$ has a unique fixed point $\beta_{4} \in X_{4}$.

Further, $A_{1}\left(\beta_{1}\right)=\beta_{2}, A_{2}\left(\beta_{2}\right)=\beta_{3}, A_{3}\left(\beta_{3}\right)=\beta_{4}, A_{4}\left(\beta_{4}\right)=\beta_{1}$

Proof: Let $x_{0}^{1}$ be an arbitrary point in $X_{1}$, let define sequence $\left\{x_{m}^{1}\right\},\left\{x_{m}^{2}\right\},\left\{x_{m}^{3}\right\}$ and $\left\{x_{m}^{4}\right\}$ in $X_{1}, X_{2}, X_{3}, X_{4}$ respectively by $\left(A_{4} A_{3} A_{2} A_{1}\right)^{m} x_{0}^{1}=x_{m}^{1}, \quad x_{m}^{2}=A_{1}\left(x_{m-1}^{1}\right), \quad x_{m}^{3}=A_{2}\left(x_{m}^{2}\right)$, $x_{m}^{4}=A_{3}\left(x_{m}^{3}\right) \quad x_{m}^{1}=A_{4}\left(x_{m}^{4}\right) \quad$ for $m=1,2,3, \ldots$ We will assume that $x_{m}^{1} \neq x_{m+1}^{1}, x_{m}^{2} \neq x_{m+1}^{2}$ and so on $x_{m}^{n} \neq x_{m+1}^{n}$ for all $\mathrm{m}$. Otherwise, if $x_{m}^{1}=x_{m+1}^{1}$ for some $\mathrm{m}$, then $x_{m}^{2}=x_{m+1}^{2}$, $x_{m}^{3}=x_{m+1}^{3}$ and $x_{m}^{4}=x_{m+1}^{4}$,we could put $x_{m}^{1}=\beta_{1}, x_{m+1}^{2}=\beta_{2}$ and $x_{m+1}^{4}=\beta_{4}$ First, we prove the sequences $\left\{x_{m}^{1}\right\},\left\{x_{m}^{2}\right\},\left\{x_{m}^{3}\right\}$ and $\left\{x_{m}^{4}\right\}$ are cauchy sequences. Taking $x^{1}=x_{m}^{1}, x^{2}=x_{m}^{2}$ in (1.1) and we obtain $M_{1}\left(x_{m}^{1}, x_{m}^{2}\right)=\left\{d_{1}^{p}\left(x_{m}^{1}, A_{4} A_{3} A_{2} x_{m}^{2}\right), d_{1}^{p}\left(x_{m}^{1}, A_{4} A_{3} A_{2} A_{1} x_{m}^{1}\right), d_{2}^{p}\left(x_{m}^{2}, A_{1} x_{m}^{1}\right)\right\}$

$=\left\{d_{1}^{p}\left(x_{m}^{1}, x_{m}^{1}\right), d_{1}^{p}\left(x_{m}^{1}, x_{m+1}^{1}\right), d_{2}^{p}\left(x_{m}^{2}, x_{m+1}^{2}\right)\right\}$

$=\left\{0, d_{1}^{p}\left(x_{m}^{1}, x_{m+1}^{1}\right), d_{2}^{p}\left(x_{m}^{2}, x_{m+1}^{2}\right)\right\}$

$$
d_{1}^{p}\left(x_{m}^{1}, x_{m+1}^{1}\right)=
$$

$d_{1}^{p}\left(A_{4} A_{3} A_{2} x_{m}^{2}, A_{4} A_{3} A_{2} A_{1} x_{m}^{1}\right)$

$\leq c \max M_{1}\left(x_{m}^{1}, x_{m}^{2}\right)+F\left(\min M_{1}\left(x_{m}^{1}, x_{m}^{2}\right)\right)$

$=c \max \left\{0, d_{1}^{p}\left(x_{m}^{1}, x_{m+1}^{1}\right), d_{2}^{p}\left(x_{m}^{2}, x_{m+1}^{2}\right)\right\}+F(0)$

$=c d_{2}^{p}\left(x_{m}^{2}, x_{m+1}^{2}\right)$

Since, $\quad$ if $\quad \max M_{1}\left(x_{m}^{1}, x_{m}^{2}\right)=d_{1}^{p}\left(x_{m}^{1}, x_{m+1}^{1}\right) \quad$,then $d_{1}^{p}\left(x_{m}^{1}, x_{m+1}^{1}\right) \leq c d_{1}^{p}\left(x_{m}^{1}, x_{m+1}^{1}\right)$ 
It follows $x_{m}^{1}=x_{m+1}^{1}$, since $0 \leq c<1$, so $d_{1}^{p}\left(x_{m}^{1}, x_{m+1}^{1}\right)$ $\leq c d_{2}^{p}\left(x_{m}^{2}, x_{m+1}^{2}\right)(1.9)$

Taking $x^{2}=x_{m}^{2}, x^{3}=x_{m-1}^{3}$ in (1.2) and (1.6), we get

$M_{2}\left(x_{m}^{2}, x_{m-1}^{3}\right)=\left\{d_{2}^{p}\left(x_{m}^{2}, A_{1} A_{4} A_{3} x_{m-1}^{3}\right), d_{2}^{p}\left(x_{m}^{2}, A_{1} A_{4} A_{3} A_{2} x_{m}^{2}\right), d_{3}^{p}\left(x_{m-1}^{3}, A_{2} x_{m}^{2}\right)\right\}$ $=\left\{d_{2}^{p}\left(x_{m}^{2}, x_{m}^{2}\right), d_{2}^{p}\left(x_{m}^{2}, x_{m+1}^{2}\right), d_{3}^{p}\left(x_{m-1}^{3}, x_{m}^{3}\right)\right\}$

$=\left\{0, d_{2}^{p}\left(x_{m}^{2}, x_{m+1}^{2}\right), d_{3}^{p}\left(x_{m-1}^{3}, x_{m}^{3}\right)\right\}$

$$
d_{2}^{p}\left(x_{m}^{2}, x_{m+1}^{2}\right)
$$

$d_{2}^{p}\left(A_{1} A_{4} A_{3} x_{m-1}^{3}, A_{1} A_{4} A_{3} A_{2} x_{m}^{2}\right)$

$\leq c \max M_{2}\left(x_{m}^{2}, x_{m-1}^{3}\right)+F\left(\min M_{2}\left(x_{m}^{2}, x_{m-1}^{3}\right)\right)$

$=c \max \left\{0, d_{2}^{p}\left(x_{m}^{2}, x_{m+1}^{2}\right), d_{3}^{p}\left(x_{m-1}^{3}, x_{m}^{3}\right)\right\}+F(0)$

Since $0 \leq c<1$, we get $d_{2}^{p}\left(x_{m}^{2}, x_{m+1}^{2}\right) \leq c d_{3}^{p}\left(x_{m-1}^{3}, x_{m}^{3}\right)$ (1.10)

Taking $x^{3}=x_{m}^{3}, x^{4}=x_{m-1}^{4}$ in (1.3) and (1.7), we get

$M_{3}\left(x_{m}^{3}, x_{m-1}^{4}\right)=\left\{d_{3}^{p}\left(x_{m}^{3}, A_{2} A_{1} A_{4} x_{m-1}^{4}\right), d_{3}^{p}\left(x_{m}^{3}, A_{2} A_{1} A_{4} A_{3} x_{m}^{3}\right), d_{4}^{p}\left(x_{m-1}^{4}, A_{3} x_{m}^{3}\right)\right\}$ $=\left\{d_{3}^{p}\left(x_{m}^{3}, x_{m}^{3}\right), d_{3}^{p}\left(x_{m}^{3}, x_{m+1}^{3}\right), d_{4}^{p}\left(x_{m-1}^{4}, x_{m}^{4}\right)\right\}$

$=\left\{0, d_{3}^{p}\left(x_{m}^{3}, x_{m+1}^{3}\right), d_{4}^{p}\left(x_{m-1}^{4}, x_{m}^{4}\right)\right\}$

$$
d_{3}^{p}\left(x_{m}^{3}, x_{m+1}^{3}\right)=d_{3}^{p}\left(A_{2} A_{1} A_{5} A_{4} x_{m-1}^{4}, A_{2} A_{1} A_{5} A_{4} A_{3} x_{m}^{3}\right)
$$

$\leq c \max M_{3}\left(x_{m}^{3}, x_{m-1}^{4}\right)+F\left(\min M_{3}\left(x_{m}^{3}, x_{m-1}^{4}\right)\right)$

$=c \max \left\{0, d_{3}^{p}\left(x_{m}^{3}, x_{m+1}^{3}\right), d_{4}^{p}\left(x_{m-1}^{4}, x_{m}^{4}\right)\right\}+F(0)$

$=c d_{4}^{p}\left(x_{m-1}^{4}, x_{m}^{4}\right)$

Replacing $\mathrm{m}$ to $\mathrm{m}-1$, we obtain

$$
d_{3}^{p}\left(x_{m-1}^{3}, x_{m}^{3}\right) \leq c d_{4}^{p}\left(x_{m-2}^{4}, x_{m-1}^{4}\right)
$$

Taking $x^{4}=x_{m}^{4}, x^{1}=x_{m-1}^{1}$ in (1.4) and (1.8), we get $M_{4}\left(x_{m}^{4}, x_{m-1}^{1}\right)=\left\{d_{4}^{p}\left(x_{m}^{4}, A_{3} A_{2} A_{1} x_{m-1}^{1}\right), d_{4}^{p}\left(x_{m}^{4}, A_{3} A_{2} A_{1} A_{4} x_{m}^{4}\right), d_{1}^{p}\left(x_{m-1}^{1}, A_{4} x_{m}^{4}\right)\right\}$

$=\left\{d_{4}^{p}\left(x_{m}^{4}, x_{m}^{4}\right), d_{4}^{p}\left(x_{m}^{4}, x_{m+1}^{4}\right), d_{1}^{p}\left(x_{m-1}^{1}, x_{m}^{1}\right)\right\}$

$=\left\{0, d_{4}^{p}\left(x_{m}^{4}, x_{m+1}^{4}\right), d_{1}^{p}\left(x_{m-1}^{1}, x_{m}^{1}\right)\right\}$

$d_{4}^{p}\left(x_{m}^{4}, x_{m+1}^{4}\right)=d_{4}^{p}\left(A_{3} A_{2} A_{1} x_{m-1}^{1}, A_{3} A_{2} A_{1} A_{4} x_{m}^{4}\right)$

$\leq c \max M_{4}\left(x_{m}^{4}, x_{m-1}^{1}\right)+F\left(\min M_{4}\left(x_{m}^{4}, x_{m-1}^{1}\right)\right)$

$=c \max \left\{0, d_{4}^{p}\left(x_{m}^{4}, x_{m+1}^{4}\right), d_{1}^{p}\left(x_{m-1}^{1}, x_{m}^{1}\right)\right\}+F(0)$

$=c d_{1}^{p}\left(x_{m-1}^{1}, x_{m}^{1}\right)$

Replacing $\mathrm{m}$ with $\mathrm{m}-2$ we obtain

$$
d_{4}^{p}\left(x_{m-2}^{4}, x_{m-1}^{4}\right) \leq c d_{1}^{p}\left(x_{m-3}^{1}, x_{m-2}^{1}\right)
$$

Using (1.9), (1.10), (1.11) and (1.12), we get $d_{1}^{p}\left(x_{m}^{1}, x_{m+1}^{1}\right) \leq c d_{2}^{p}\left(x_{m}^{2}, x_{m+1}^{2}\right) \leq c^{2} d_{3}^{p}\left(x_{m-1}^{3}, x_{m}^{3}\right) \leq$ $c^{3} d_{4}^{p}\left(x_{m-2}^{4}, x_{m-1}^{4}\right) \leq c^{4} d_{1}^{p}\left(x_{m-3}^{1}, x_{m-2}^{1}\right)$

$\leq \ldots \leq c^{8} d_{1}^{p}\left(x_{m-6}^{1}, x_{m-5}^{1}\right)$

$\leq \ldots \leq\left\{\begin{array}{l}c^{4 k} d_{1}^{p}\left(x_{1}^{1}, x_{2}^{1}\right), m=3 k+1 \\ c^{4 k} d_{1}^{p}\left(x_{0}^{1}, x_{1}^{1}\right), m=3 k\end{array}\right\}$
Since $0 \leq c<1$, the sequences $\left\{x_{m}^{1}\right\}$, $\left\{x_{m}^{1}\right\},\left\{x_{m}^{2}\right\},\left\{x_{m}^{3}\right\},\left\{x_{m}^{4}\right\}$ are cauchy sequences. Since $\left(X_{i}, d_{i}\right)$ be complete metric spaces where $i=1,2,3,4$

$\lim _{m \rightarrow \infty} x_{m}^{1}=\beta_{1} \in X_{1}, \lim _{m \rightarrow \infty} x_{m}^{2}=\beta_{2} \in X_{2}$,

$\lim _{m \rightarrow \infty} x_{m}^{3}=\beta_{3} \in X_{3}, \lim _{m \rightarrow \infty} x_{m}^{4}=\beta_{4} \in X_{4}$

Now taking $x^{1}=x_{m}^{1}$ and $x^{2}=\beta_{2}$ in the inequality (1.5) we obtain

$$
\begin{aligned}
& d_{1}^{p}\left(A_{4} A_{3} A_{2} \beta_{2}, x_{m+1}^{1}\right)=d_{1}^{p}\left(A_{4} A_{3} A_{2} \beta_{2}, A_{4} A_{3} A_{2} A_{1} x_{m}^{1}\right) \\
\leq & c \max M_{1}\left(x_{m}^{1}, \beta_{2}\right)+F\left(\min M_{1}\left(x_{m}^{1}, \beta_{2}\right)\right)
\end{aligned}
$$

Where

$M_{1}\left(x_{m}^{1}, \beta_{2}\right)=\left\{d_{1}^{p}\left(x_{m}^{1}, A_{4} A_{3} A_{2} \beta_{2}\right)\right.$,

$\left.d_{1}^{p}\left(x_{m}^{1}, A_{4} A_{3} A_{2} A_{1} x_{m}^{1}\right), d_{2}^{p}\left(\beta_{2}, A_{1} x_{m}^{1}\right)\right\}$

$=\left\{d_{1}^{p}\left(x_{m}^{1}, A_{5} A_{4} A_{3} A_{2} \beta_{2}\right), d_{1}^{p}\left(x_{m}^{1}, x_{m+1}^{1}\right), d_{2}^{p}\left(\beta_{2}, x_{m+1}^{2}\right)\right\}$

As $\mathrm{m}$ tend to infinity in (1.13) and $\mathrm{F}$ is continuous at 0 we get $d_{1}^{p}\left(A_{4} A_{3} A_{2} \beta_{2}, \beta_{1}\right) \leq c d_{1}^{p}\left(\beta_{1}, A_{4} A_{3} A_{2} \beta_{2}\right) \quad, \quad$ So $\quad$ we get, $A_{4} A_{3} A_{2} \beta_{2}=\beta_{1}$

In same way, we obtain, $A_{1} A_{4} A_{3} \beta_{3}=\beta_{2}, A_{2} A_{1} A_{4} \beta_{4}=\beta_{3}, A_{3} A_{2} A_{1} \beta_{1}=\beta_{4}$

Using (1.5), taking $x^{1}=\beta_{1}, x^{2}=x_{m}^{2} \quad$ we get $\quad d_{1}^{p}\left(x_{m}^{1}, A_{4} A_{3} A_{2} A_{1} \beta_{1}\right)=d_{1}^{p}\left(A_{4} A_{3} A_{2} x_{m}^{2}, A_{4} A_{3} A_{2} A_{1} \beta_{1}\right)$ $\leq c \max M_{1}\left(\beta_{1}, x_{m}^{2}\right)+F\left(\min M_{1}\left(\beta_{1}, x_{m}^{2}\right)\right)$

Where, $M_{1}\left(\beta_{1}, x_{m}^{2}\right)=\left\{d_{1}^{p}\left(\beta_{1}, A_{4} A_{3} A_{2} x_{m}^{2}\right)\right.$, $\left.d_{1}^{p}\left(\beta_{1}, A_{4} A_{3} A_{2} A_{1} \beta_{1}\right), d_{2}^{p}\left(x_{m}^{2}, A_{1} \beta_{1}\right)\right\}$ $=\left\{d_{1}^{p}\left(\beta_{1}, x_{m}^{1}\right), d_{1}^{p}\left(\beta_{1}, A_{4} A_{3} A_{2} A_{1} \beta_{1}\right), d_{2}^{p}\left(x_{m}^{2}, A_{1} \beta_{1}\right)\right\}$

Now letting $\mathrm{m}$ tend to infinity we get

$d_{1}^{p}\left(\beta_{1}, A_{4} A_{3} A_{2} A_{1} \beta_{1}\right)$

$\leq c \max \left\{d_{1}^{p}\left(\beta_{1}, \beta_{1}\right), d_{1}^{p}\left(\beta_{1}, A_{4} A_{3} A_{2} A_{1} \beta_{1}\right), d_{2}^{p}\left(\beta_{2}, A_{1} \beta_{1}\right)\right\}$ $=c \max \left\{d_{1}^{p}\left(\beta_{1}, A_{4} A_{3} A_{2} A_{1} \beta_{1}\right), d_{2}^{p}\left(\beta_{2}, A_{1} \beta_{1}\right)\right\}$

From which it follows, or $d_{1}^{p}\left(\beta_{1}, A_{4} A_{3} A_{2} A_{1} \beta_{1}\right)$ $\leq c d_{1}^{p}\left(\beta_{1}, A_{4} A_{3} A_{2} \beta_{1}\right) \Leftrightarrow A_{4} A_{3} A_{2} A_{1} \beta_{1}=\beta_{1}$ or $d_{1}^{p}\left(\beta_{1}, A_{4} A_{3} A_{2} A_{1} \beta_{1}\right) \leq c d_{2}^{p}\left(\beta_{2}, A_{1} \beta_{1}\right)$. This can be also written in following form

$$
d_{1}^{p}\left(\beta_{1}, A_{4} \beta_{4}\right) \leq c d_{2}^{p}\left(\beta_{2}, A_{1} \beta_{1}\right)
$$

Since, $A_{4} A_{3} A_{2} A_{1} \beta_{1}=\beta_{n}$, Taking $x^{3}=x_{m}^{3}, x^{2}=\beta_{2}$ in inequality (1.6) in the same way as above, we obtain $d_{2}^{p}\left(x_{m+1}^{2}, A_{1} A_{4} A_{3} A_{2} \beta_{2}\right)=d_{2}^{p}\left(A_{1} A_{4} A_{3} x_{m}^{3}, A_{1} A_{4} A_{3} A_{2} \beta_{2}\right)$ $\leq c \max M_{2}\left(\beta_{2}, x_{m}^{3}\right)+F\left(\min M_{2}\right) \quad$ where, $M_{2}\left(\beta_{2}, x_{m}^{3}\right)$ $\left\{d_{2}^{p}\left(\beta_{2}, A_{1} A_{4} A_{3} x_{m}^{3}\right), d_{2}^{p}\left(\beta_{2}, A_{1} A_{4} A_{3} A_{2} \beta_{2}\right), d_{3}^{p}\left(x_{m}^{3}, A_{2} \beta_{2}\right)\right\}$ $=\left\{d_{2}^{p}\left(\beta_{2}, x_{m+1}^{3}\right), d_{2}^{p}\left(\beta_{2}, A_{1} A_{4} A_{3} A_{2} \beta_{2}\right), d_{3}^{p}\left(x_{m}^{3}, A_{2} \beta_{2}\right)\right\}$

Now letting $\mathrm{m}$ tend to infinity we get $d_{2}^{p}\left(\beta_{2}, A_{1} A_{4} A_{3} A_{2} \beta_{2}\right) \leq c \max \left\{d_{2}^{p}\left(\beta_{2}, \beta_{2}\right), d_{2}^{p}\left(\beta_{2}, A_{1} A_{4} A_{3} A_{2}\right.\right.$ 
From which it follows, Then,

$d_{2}^{p}\left(\beta_{2}, A_{1} A_{4} A_{3} A_{2} \beta_{2}\right) \leq c d_{2}^{p}\left(\beta_{2}, A_{1} A_{4} A_{3} A_{2} \beta_{2}\right) \Leftrightarrow A_{1} A_{4} A_{3} A_{2} \beta_{2}=\beta_{2}$

$$
\text { or } d_{2}^{p}\left(\beta_{2}, A_{1} \beta_{1}\right) \leq c d_{3}^{p}\left(\beta_{3}, A_{2} \beta_{2}\right)
$$

Continuously like above, we get

$d_{3}^{p}\left(\beta_{3}, A_{2} \beta_{2}\right) \leq c d_{4}^{p}\left(\beta_{4}, A_{3} \beta_{3}\right), d_{4}^{p}\left(\beta_{4}, A_{3} \beta_{3}\right) \leq c d_{1}^{p}\left(\beta_{1}, A_{4} \beta_{4}\right)$,
By
(1.14),
(1.15),
we
obtain $d_{1}^{p}\left(\beta_{1}, A_{5} \beta_{5}\right) \leq c d_{2}^{p}\left(\beta_{2}, A_{1} \beta_{1}\right)$

$$
d_{2}^{p}\left(\beta_{2}, A_{1} \beta_{1}^{1}\right) \leq c d_{3}^{p}\left(\beta_{3}, A_{2} A_{1} \beta_{1}^{1}\right)
$$

Continuously like above, using (1.7), (1.8), we get

$d_{3}^{p}\left(\beta_{3}, A_{2} A_{1} \beta_{1}^{1}\right) \leq c d_{4}^{p}\left(\beta_{4}, A_{3} A_{2} A_{1} \beta_{1}^{1}\right), d_{4}^{p}\left(\beta_{4}, A_{3} A_{2} A_{1} \beta_{1}^{1}\right) \leq c d_{1}^{p}\left(\beta_{1}^{1}, \beta_{1}\right)$

$\leq c^{2} d_{3}^{p}\left(\beta_{3}, A_{2} \beta_{2}\right) \leq c^{3} d_{4}^{p}\left(\beta_{4}, A_{3} \beta_{3}\right) \leq c^{4} d_{1}^{p}\left(\beta_{1}, A_{4} \beta_{4}\right)$

$\Rightarrow d_{1}^{p}\left(\beta_{1}, A_{5} \beta_{5}\right) \leq c^{4} d_{1}^{p}\left(\beta_{1}, A_{4} \beta_{4}\right)$

$\Leftrightarrow A_{5} \beta_{5}=\beta_{1} \quad$, since $0 \leq c<1 \quad$, Thus again

$d_{1}^{p}\left(\beta_{1}, A_{4} A_{3} A_{2} A_{1} \beta_{1}\right)=d_{1}^{p}\left(\beta_{1}, A_{4} \beta_{4}\right)=0$

$\Leftrightarrow A_{4} A_{3} A_{2} A_{1} \beta_{1}=\beta_{1}$ So, we proved that $\beta_{1}$ is a fixed point of $A_{4} A_{3} A_{2} A_{1} \quad ; \quad A_{4} A_{3} A_{2} A_{1}$ has fixed point $\beta_{1} \in X_{1} ; A_{1} A_{4} A_{3} A_{2}$ has fixed point $\beta_{2} \in X_{2}, A_{2} A_{1} A_{4} A_{3}$ has fixed point $\beta_{3} \in X_{3}$, and $A_{3} A_{2} A_{1} A_{4}$ has fixed point $\beta_{4} \in X_{4} \quad$. Further, we also showed that $A_{1}\left(\beta_{1}\right)=\beta_{2}, A_{2}\left(\beta_{2}\right)=\beta_{3}, A_{3}\left(\beta_{3}\right)=\beta_{4}, A_{4}\left(\beta_{4}\right)=\beta_{1}$

Now let assume now that $\beta_{1}{ }_{1} \in X_{1}$ is another fixed point of $A_{4} A_{3} A_{2} A_{1}$, different from $\beta_{1}$.

Using (1.5), if we take $x^{1}=\beta_{1}, x^{2}=A_{1} \beta_{1}^{1}$, we get $d_{1}^{p}\left(\beta_{1}^{1}, \beta_{1}\right)=d_{1}^{p}\left(A_{4} A_{3} A_{2} A_{1} \beta_{1}^{1}, A_{4} A_{3} A_{2} A_{1} \beta_{1}\right)$

$\leq c \max M_{1}\left(\beta_{1}, A_{1} \beta_{1}^{1}\right)+F\left(\min M_{1}\left(\beta_{1}, A_{1} \beta_{1}^{1}\right)\right)$

Where

$M_{1}\left(\beta_{1}, A_{1} \beta_{1}^{1}\right)=\left\{d_{1}^{p}\left(\beta_{1}, A_{4} A_{3} A_{2} A_{1} \beta_{1}^{1}\right), d_{1}^{p}\left(\beta_{1}, A_{4} A_{3} A_{2} A_{1} \beta_{1}\right), d_{2}^{p}\left(A_{1} \beta_{1}^{1}, A_{1} \beta_{1}\right)\right\}$

$=\left\{d_{1}^{p}\left(\beta_{1}, \beta_{1}^{1}\right), d_{1}^{p}\left(\beta_{1}, \beta_{1}\right), d_{2}^{p}\left(A_{1} \beta_{1}^{1}, \beta_{2}\right)\right\}$

From which it follows

$$
d_{1}^{p}\left(\beta_{1}^{1}, \beta_{1}\right) \leq c d_{2}^{p}\left(A_{1} \beta_{1}^{1}, \beta_{2}\right)
$$

Taking $x^{3}=\beta_{3}, x^{2}=A_{1} \beta_{1}^{1}$ in inequality (1.6) we obtain $d_{2}^{p}\left(\beta_{2}, A_{1} \beta_{1}^{1}\right)=d_{2}^{p}\left(A_{1} A_{4} A_{3} \beta_{3}, A_{1} A_{3} A_{2} A_{1} \beta_{1}^{1}\right)$ $\leq c \max M_{2}\left(A_{1} \beta_{1}^{1}, \beta_{3}\right)+F\left(\min M_{2}\left(A_{1} \beta_{1}^{1}, \beta_{3}\right)\right)$

Where,

$M_{2}\left(A_{1} \beta_{1}^{1}, \beta_{3}\right)=\left\{d_{2}^{p}\left(A_{1} \beta_{1}^{1}, A_{1} A_{4} A_{3} \beta_{3}\right), d_{2}^{p}\left(A_{1} \beta_{1}^{1}, A_{1} A_{4} A_{3} A_{2} A_{1} \beta_{1}^{1}\right), d_{3}^{p}\left(\beta_{3}, A_{2} A_{1} \beta_{1}^{1}\right)\right\}$ $\left.=d_{2}^{p}\left(A_{1} \beta_{1}^{1}, \beta_{2}\right), d_{2}^{p}\left(A_{1} \beta_{1}^{1}, A_{1} \beta_{1}^{1}\right), d_{3}^{p}\left(\beta_{3}, A_{2} A_{1} \beta_{1}^{1}\right)\right\}$

By using (1.16), (1.17) and (1.18)

$$
d_{1}^{p}\left(\beta_{1}^{1}, \beta_{1}\right) \quad \leq c d_{2}^{p}\left(A_{1} \beta_{1}^{1}, \beta_{2}\right)
$$

$\leq c^{2} d_{3}^{p}\left(\beta_{3}, A_{2} A_{1} \beta_{1}^{1}\right) \leq c^{3} d_{4}^{p}\left(A_{3} A_{2} A_{1} \beta_{1}^{1}, \beta_{4}\right) \leq$

$c^{4} d_{1}^{p}\left(\beta_{1}^{1}, \beta_{1}\right) \quad \Leftrightarrow d_{1}^{p}\left(\beta_{1}^{1}, \beta_{1}\right) \leq c^{4} d_{1}^{p}\left(\beta_{1}^{1}, \beta_{1}\right) \quad$ where $0 \leq c<1$. It follows that $\beta_{1}^{1}=\beta_{1}$.

Thus we proved $\beta_{1}$ is the fixed point of $A_{4} A_{3} A_{2} A_{1}$. In the same way, it can be shown that $A_{4} A_{3} A_{2} A_{1}$ has a unique fixed point $\beta_{1} \in X_{1}, A_{1} A_{4} A_{3} A_{2}$ has a unique fixed point $\beta_{2} \in X_{2}, A_{2} A_{1} A_{4} A_{3}$ has a unique fixed point $\beta_{3} \in X_{3}$ and $A_{3} A_{2} A_{1} A_{4}$ has a unique fixed point $\beta_{4} \in X_{4}$.

\section{ACKNOWLEDGEMENTS}

It is with a feeling of great pleasure that we would like to express my most sincere heartfelt gratitude to Chancellor of M.M. University, Haryana (INDIA), Shri Tarsem Garg and Head of the Mathematics Dept. Prof. Deepak Gupta for providing us the necessary facilities in the department.

\section{REFERENCES}

[1] L. Kikina, "Fixed point theorem in three metric spaces," Int.Journal of math.analysis, vol. 3, no. 13, pp. 619-626, 2009.

[2] S. Č. Nešić, "A fixed point theorem in two metric spaces," Bull. Math. Soc. Sci. Math. Roumanie(N.S.)., vol. 44, no. 92, pp. 253-257, 2001.

[3] B. Fisher, "Fixed points theorem on two metric spaces," Glasnik Mat., vol. 16 , no. 36 , pp. 333-337, 1981.

[4] R. K. Jain, H. K. Sahu, and B. Fisher, "Related fixed point theorems for three metric spaces," Novi Sad J. Math., vol. 26, no. 1, pp.11-17, 1996.

[5] V. Popa, "Fixed points on two metric spaces," Zb. Rad, Prirod, Mat. Fak(N.S.)Ser. Mat.,vol. 21, pp. 83-93, 1991. 